\title{
Pacific
}

Journal of

Mathematics

\section{IMMERSIONS UP TO JOINT-BORDISM}

\author{
GUI SONG LI
}




\title{
IMMERSIONS UP TO JOINT-BORDISM
}

\author{
GUI-SONG LI
}

A necessary and sufficient condition for a map to be joint-bordant to an immersion is given in terms of StiefelWhitney numbers.

1. Introduction. This note is devoted to a study of immersions of manifolds into manifolds up to joint-bordism. We will work throughout in the category of smooth manifolds and smooth maps.

A map of dimension $(n, k)$ is a map of a closed $n$-manifold into a closed $(n+k)$-manifold. Two maps $f_{0}: M_{0} \rightarrow N_{0}$ and $f_{1}: M_{1} \rightarrow N_{1}$ of dimension $(n, k)$ are said to be joint-bordant if there is a map $F: V \rightarrow W$ extending $f_{0} \cup f_{1}$ where $V$ and $W$ are compact manifolds with $\partial V=M_{0} \cup M_{1}$ and $\partial W=N_{0} \cup N_{1}$. Joint-bordism classes of maps of dimension $(n, k)$ form an abelian group under the disjoint union which we denote by $M(n, k)$. It is well known that Stiefel-Whitney numbers form a complete system of invariants for the joint-bordism theory [9]. So one may hope to characterize maps joint-bordant to immersions or embeddings in terms of these numbers whenever $k>0$. For the case of embeddings this has already been settled by Brown [3]; his proof is based on a construction suggested by Stong. In this note, using the model construction of Koschorke [6], we shall give such a criterion for maps joint-bordant to immersions in the "metastable" range $n \leq 2 k$.

Our method of proof can also be applied to study immersions up to various oriented joint-bordism relations. These are naturally defined for the following restricted classes of maps (see Stong [9]):

$C_{1}$ : maps with oriented source manifolds;

$C_{2}$ : maps with oriented target manifolds;

$C_{3}$ : maps with oriented stable normal bundles;

$C_{4}$ : maps with oriented source and target manifolds. 
In fact, let $M_{r}(n, k)$ be the resulting oriented joint-bordism group of maps of dimension $(n, k)$ belonging to $C_{r}$ and let $\rho_{r}: M_{r}^{(n, k)} \rightarrow$ $M(n, k)$ be the natural forgetful homomorphism. Then $x \in M_{r}(n, k)$ contains an immersion if $\rho_{r}(x)$ does provided $n \leq 2 k$.

We now summarize our main results by the following

MAIN TheOREM. Let $n \leq 2 k$. Then under all five orientedness assumptions above a map $f: M \rightarrow N$ of dimension $(n, k)$ is jointbordant to an immersion if and only if

$$
w_{m}(f) f^{*} w_{\mu_{1}}(N) w_{\mu_{2}}(M) \cap[M]=0
$$

for all $m>k$ and all partions $\mu_{1}, \mu_{2}$ with $\left|\mu_{1}\right|+\left|\mu_{2}\right|=n-m$. Here $w(f)=f^{*} w(N) \bar{w}(M)$ is the total Stiefel-Whitney class of the stable normal bundle of $f$ and $[M]$ is the $\mathbf{Z}_{2}$-fundamental class of $M$.

REMARK. Stong [9] showed that the unoriented joint-bordism class of $f$ is completely determined by Stiefel-Whitney numbers of the form

$$
w_{\mu_{1}}(N) f_{!} w_{\mu_{2}}(M) \cdots f_{!} w_{\mu_{l}}(M) \cap[N]
$$

which, in the case of $l>1$, are equal to

$$
f^{*} w_{\mu_{1}}(N) w_{\mu_{2}}(M) f^{*} f_{!} w_{\mu_{3}}(M) \cdots f^{*} f_{!} w_{\mu_{l}}(M) \cap[M]
$$

Here $\mu_{i}$ are partitions with $\sum\left|\mu_{i}\right|=n-(l-2) k$ and $f_{!}: H^{*}\left(M, \mathbf{Z}_{2}\right) \rightarrow$ $H^{*+k}\left(N, \mathbf{Z}_{2}\right)$ is the $U m k e h r$ homomorphism defined by taking a cohomology class $x$ into the Poincaré dual of $f_{*}(x \cap[M])$. In the case of $n \leq 2 k$ the $f^{*} f_{!} w_{\mu_{i}}(M)$ factors above disappear if $\left|\mu_{1}\right|+\left|\mu_{2}\right|>k$. So our main theorem is equivalent to saying that: "if $n \leq 2 k$ then under all five orientedness assumptions above $f$ is joint-bordant to an immersion if and only if all Stiefel-Whitney numbers of the form $\left(^{*}\right)$ involving $w_{m}(f)$ where $m>k$ are zero." I do not know whether this statement remains true if $n>2 k$.

REMARK. It was conjectured by Olk [8] that in a certain "metastable" range (probably $n \leq 2 k-1$ ) a closed $n$-manifold $M$ can be immersed into $\mathbb{R}^{n+k}$ up to bordism, or equivalently, a map $f: M \rightarrow$ $\mathbb{S}^{n+k}$ is bordant in the sense of Atiyah [1] to an immersion if and only if

$$
\bar{w}_{m}(M) w_{\mu}(M) \cap[M]=0
$$


for all $m>k$ and all partions $\mu$ of $n-m$. By our results, these numbers are zero if and only if $f$ is joint-bordant in $M_{2}(n, k)$ (even in $M_{4}(n, k)$ if $M$ is oriented) to an immersion provided $n \leq 2 k$. Olk [8] showed that the above statement is always true if $k \geq n-7$. So in these cases "joint-bordant to an immersion" and "bordant to an immersion" are the same for a map into a sphere.

I would like to thank the Alexander von Humboldt-Stiftung for financial support and Ulrich Koschorke for his hospitality. I also want to thank M.A. Aguilar for drawing my attention to the work of R.L.W. Brown.

2. The unoriented case. We start by describing the behavior of a generic map around its singularity subset. Recall that a map is generic if its self-intersections are transversal and its jet sections are transversal to the Boardman manifolds [2]. Given a generic map $f: M \rightarrow N$ of dimension $(n, k)$, we denote by $\Sigma_{f}$ the set of singular points of $f$ and by $\bar{\Sigma}_{f}=f\left(\Sigma_{f}\right)$ its image. Hereafter we always assume $k>0$. In the case of $n \leq 2 k+2, \quad \Sigma_{f}$ is a closed submanifold of dimension $n-k-1$ containing only points over which $d f$ is of rank $n-1, \quad f$ is an embedding restricted to $\Sigma_{f}$ and the bordism class of $\Sigma_{f}$ depends only on the joint-bordism class of $f$.

Let us denote by Ker and Coker the 1 - and $(k+1)$-dimensional vector bundles over $\Sigma_{f}$ and $\bar{\Sigma}_{f}$ which are determined by kernels and cokernels of $d f \mid \Sigma_{f}$ respectively. By Koschorke [6] the normal bundle $\nu$ of $\Sigma_{f}$ in $M$ is canonically isomorphic to Ker $\otimes$ Coker. Here and henceforth we shall always omit the pull-backs of vector bundles by $f \mid \Sigma_{f}$. Now let $\bar{\nu}$ be the normal bundle of $\bar{\Sigma}_{f}$ in $N$ and let $\bar{f}: E \nu \rightarrow E \bar{\nu}$ be the map between total spaces of normal bundles determined by the $k$-morphism $\nu \rightarrow \bar{\nu}$ induced by $d f$. Then by applying the model construction given in Section 1 of Koschorke [6] to the bundle homomorphism $d f \mid \Sigma_{f}$ of constant rank $n-1$, we obtain a nondegenerate $(n-1)$-morphism $T(E \nu) \rightarrow T(E \bar{\nu})$ covering $\bar{f}$ with $\Sigma_{f}$ its singularity subset, and hence a generic map

$$
\alpha_{f}: E \nu \rightarrow E \bar{\nu}
$$

with $\Sigma_{f}$ its singularity subset by Feit [5]. Moreover, suitably identifying $E \nu$ and $E \bar{\nu}$ with certain tubular neighborhoods of $\Sigma_{f}$ in $M$ 
and of $\bar{\Sigma}_{f}$ in $N$ respectively, we can identify $\alpha_{f}$ with $f$ restricted to a small tubular neighborhood of $\Sigma_{f}$ in $M$ (see [6]).

Now let $x \in M(n, k)$ be represented by a generic map $f: M \rightarrow N$ and let $n \leq 2 k+2$. We denote by

$$
\beta_{f}: \Sigma_{f} \rightarrow B O_{k+1} \times \mathbb{P}^{\infty}
$$

the map defined by the classifying maps of Coker and Ker. The bordism obstruction $\Phi(x)$ is defined to be the unoriented bordism class of $\beta_{f}$. It is readily seen that

$$
\Phi: M(n, k) \rightarrow \Re_{n-k-1}\left(B O_{k+1} \times \mathbb{P}^{\infty}\right)
$$

is a well defined homomorphism.

THEOREM . If $n \leq 2 k$ then $x \in M(n, k)$ contains an immersion if and only if $\Phi(x)=0$.

Proof. If $x$ contains an immersion then clearly $\Phi(x)=0$. To prove the contrary let $f: M \rightarrow N$ be a generic map of dimension $(n, k)$ so that $\beta_{f}$ is null-bordant. We have to show that $f$ is joint-bordant to an immersion.

In the dimensional range $n \leq 2 k+1$ there is a $k$-dimensional vector bundle $\nu_{0}$ over $\Sigma_{f}$ so that $\nu \cong \nu_{0} \oplus \mathrm{Ker}$. In this case it is easy to see that $\bar{\nu} \cong \nu_{0} \oplus$ Coker and that the $k$-morphism $\nu \rightarrow \bar{\nu}$ induced by $d f$ then corresponds to the $k$-morphism

$$
\nu_{0} \oplus \text { Ker } \rightarrow \nu_{0} \oplus \text { Coker }
$$

defined by $a \oplus b \rightarrow a$. Let us denote by $\lambda$ and $\gamma$ the canonical vector bundles over $\mathbb{P}^{\infty}$ and $B O_{k+1}$ respectively, and by $G: \Sigma \rightarrow$ $B O_{k+1} \times \mathbb{P}^{\infty}$ a null-bordism of $\beta_{f}$. For dimensional reasons $\nu_{0}$, which is stably isomorphic to $G^{*} \lambda \otimes G^{*} \gamma-G^{*} \lambda$ restricted over $\Sigma_{f}$, can be extended to a $k$-dimensional vector bundle $\eta_{0}$ over $\Sigma$ so that $G^{*} \lambda \otimes G^{*} \gamma \cong \eta_{0} \oplus G^{*} \lambda$. Now let $\eta=G^{*} \lambda \otimes G^{*} \gamma$ and let $\bar{\eta}=\eta_{0} \oplus G^{*} \gamma$. Then $\eta$ and $\bar{\eta}$ restrict to $\nu$ and $\bar{\nu}$ over $\Sigma_{f}$ respectively, and the $k$ morphism

$$
\eta \cong \eta_{0} \oplus G^{*} \lambda \rightarrow \eta_{0} \oplus G^{*} \gamma \cong \bar{\eta}
$$

defined by $a \oplus b \rightarrow a$ restricts to the $k$-morphism $\nu \rightarrow \bar{\nu}$ induced by $d f$. Applying the model construction of Koschorke to the obvious 
bundle homomorphism $\eta \oplus T \Sigma_{f} \rightarrow \bar{\eta} \oplus T \Sigma_{f}$ of constant rank $n-1$, we obtain a generic map

$$
F: E \eta \rightarrow E \bar{\eta}
$$

with $\Sigma_{f}$ its singularity subset, which, after identifying $\Sigma_{f} \subset E \bar{\eta}$ with $\bar{\Sigma}_{f}$ via $f$, is readily seen to be an extension of the map $\alpha_{f}$.

Now let $M_{1}$ be defined by glueing $M-\operatorname{int} T$ and a sphere bundle $S \eta$ of $\eta$ along the boundaries via a diffeomorphism between $\partial T$ and $S \nu$ where $T$ is a small tubular neighborhood of $\Sigma_{f}$ in $M$, and let $N_{1}$ be defined similarly. Then $M_{1}$ and $N_{1}$ are closed smooth manifolds after straightening possible angles. Moreover, if the above constructions are suitably done, then $f$ and $F$ can be fitted to yield an immersion $f_{1}: M_{1} \rightarrow N_{1}$ which is readily seen to be joint-bordant to $f$. This completes the proof of the theorem.

We now calculate the Stiefel-Whitney numbers of the map $\beta_{f}$ : $\Sigma_{f} \rightarrow B O_{k+1} \times \mathbb{P}^{\infty}$ associated to a given generic map $f: M \rightarrow N$ of dimension $(n, k)$. By definition the Stiefel-Whitney numbers of $\beta_{f}$ take the form

$$
w_{\mu_{1}}(\text { Ker }) w_{\mu_{2}}(\text { Coker }) w_{\mu_{3}}\left(\Sigma_{f}\right) \cap\left[\Sigma_{f}\right]
$$

where $\mu_{i}$ are partitions with $\sum\left|\mu_{i}\right|=n-k-1$. We have the following simple relations among total Stiefel-Whitney classes:

$$
\begin{gathered}
w(\text { Ker }) w(\text { Coker })=\left.w(f)\right|_{\Sigma_{f}} \\
w(\text { Ker } \otimes \text { Coker }) w\left(\Sigma_{f}\right)=\left.w(M)\right|_{\Sigma_{f}} .
\end{gathered}
$$

It follows that every Stiefel-Whitney number of $\beta_{f}$ is the sum of numbers of the form

$$
\left.w_{1}(\mathrm{Ker})^{m}\left(f^{*} w_{\mu_{1}^{\prime}}(N) w_{\mu_{2}^{\prime}}(M)\right)\right|_{\Sigma_{f}} \cap\left[\Sigma_{f}\right]
$$

which by 9.11 of Koschorke [6] are equal to

$$
w_{m+k+1}(f) f^{*} w_{\mu_{1}^{\prime}}(N) w_{\mu_{2}^{\prime}}(M) \cap[M]
$$

This proves our main theorem for the unoriented case. 
3. Oriented cases. We now extend the argument above to study various oriented cases. For our purpose we shall need the notion of oriented bordism group with coefficients. Let $\phi$ be a vector bundle over $X$, we denote by $\bar{\Omega}_{n}(X, \phi)$ the bordism group of triples $(M, f$, or ) where $M$ is a closed $n$-manifold, $f: M \rightarrow X$ is a map and or is an isomorphism between orientation line bundles of $T M$ and $f^{*} \phi$. This kind of bordism groups was first studied by Atiyah [1] and then by Koschorke [6]. Our notational convention follows from that of Koschorke. It should be noticed that $\bar{\Omega}_{*}(X, \phi) \cong \Omega_{*}(X)$ if $\phi$ is orientable and that

$$
\bar{\Omega}_{*}\left(X \times \mathbb{P}^{\infty}, \lambda+\phi\right) \cong \Re_{*}(X) .
$$

The bordism group $\bar{\Omega}_{*}(X, \phi)$ has several properties analogous to the usual oriented bordism group, including a generalization of Rochlin's theorem given in [4].

Similar to the unoriented case, if $n \leq 2 k+2$ then for each $1 \leq$ $r \leq 4$ we can define a homomorphism $\phi_{r}: M_{r}(n, k) \rightarrow B_{r}$ where

$$
\begin{aligned}
& B_{1}=\bar{\Omega}_{n-k-1}\left(B O_{k+1} \times \mathbb{P}^{\infty}, \lambda \otimes \gamma_{k+1}\right), \\
& B_{2}=\bar{\Omega}_{n-k-1}\left(B O_{k+1} \times \mathbb{P}^{\infty}, \lambda \otimes \gamma_{k+1}+\gamma_{k+1}-\lambda\right), \\
& B_{3}=\Re_{n-k-1}\left(B S O_{l} \times \mathbb{P}^{\infty}\right), \quad l \gg 0, \\
& B_{4}=\bar{\Omega}_{n-k-1}\left(B S O_{l} \times \mathbb{P}^{\infty}, \lambda \otimes\left(\widetilde{\gamma}_{l}-\mathbb{R}^{l-k-1}\right)\right), \quad l \gg 0,
\end{aligned}
$$

$\gamma_{k+1}$ and $\tilde{\gamma}_{l}$ are canonical vector bundles over $B O_{k+1}$ and $B S O_{l}$ respectively. To see this notice that if $f$ is a generic map belonging to the class $C_{r}$ then the map $\beta_{f}$ defined in Section 1 also represents a bordism class of $B_{r}$. For example, if $r=3$ then in the definition of $\beta_{f}$ the bundle Coker - Ker can be canonically oriented. Let $\tau$ : $B O_{k+1} \times \mathbb{P}^{\infty} \rightarrow B O_{l} \times \mathbb{P}^{\infty}$ be defined by the classifying map of $\gamma_{k+1}-\lambda$ and $\lambda$. Then $\tau \circ \beta_{f}$ can be lifted to a map into $B S O_{l} \times \mathbb{P}^{\infty}$ which represents a bordism class of $B_{3}$.

Now, a similar argument as in the unoriented case leads to the following

THEOREM . If $n \leq 2 k$ then for each $1 \leq r \leq 4$ a joint-bordism class $x \in M_{r}(n, k)$ contains an immersion if and only if $\Phi_{r}(x)=0$.

REMARK. In [7] we defined a normal bordism obstruction for a map to be bordant in the sense of Atiyah to an immersion as 
well as its various oriented versions. Since the invariants $\Phi_{r}$ here are images under certain forgetful homomorphisms and projections of our invariants in [7], the images of $\Phi_{r}$ contain only 2-primary torsion elements. Later we will see that all these elements are in fact of order 2 .

We now proceed to prove our main theorem for the remaining four oriented cases. In what follows, when $l$ is large enough, $B(S) O_{l}$ and $\gamma_{l}$ will be simply denoted by $B(S) O$ and $\gamma$ respectively.

If $r=1$ and $k$ is even, or if $r=2$ and $k$ is odd, or if $r=3$, or if $r=4$ and $k$ is even, then $B_{r}$ is canonically isomorphic to $\Re_{n-k-1}(B(S) O)$ or to $\Re_{n-k-1}\left(B S O \times \mathbb{P}^{\infty}\right)$. In these cases, our result follows by calculating characteristic numbers as in the unoriented case.

If $r=2$ and $k$ is even, or if $r=4$ and $k$ is odd, then $B_{r}$ is canonically isomorphic to $\Omega_{n-k-1}\left(B(S) O \times \mathbb{P}^{\infty}\right)$ which contains only $\mathbb{Z}$ and $\mathbb{Z}_{2}$ factors. It follows that $\Phi_{r}(x)$ is an element of order 2 and hence is determined by its Stiefel-Whitney numbers (see [4]). In these cases, our result can again be proved by calculating characteristic numbers.

It remains to consider the case when $r=1$ and $k$ is odd. Since $B_{1} \cong \bar{\Omega}_{n-k-1}\left(B O \times \mathbb{P}^{\infty}, \gamma\right)$ if $k$ is odd and since $\Phi_{1}(x)$ is a 2-primary torsion by our last remark, it suffices to show that the natural forgetful homomorphism

$$
\rho: \bar{\Omega}_{*}\left(B O \times \mathbb{P}^{\infty}, \gamma\right) \rightarrow \Re_{*}\left(B O \times \mathbb{P}^{\infty}\right)
$$

is injective restricted to the 2-primary torsion subgroup of $\bar{\Omega}_{*}(B O \times$ $\left.\mathbb{P}^{\infty}, \gamma\right)$. For this purpose we fit $\rho$ into the following commutative diagram

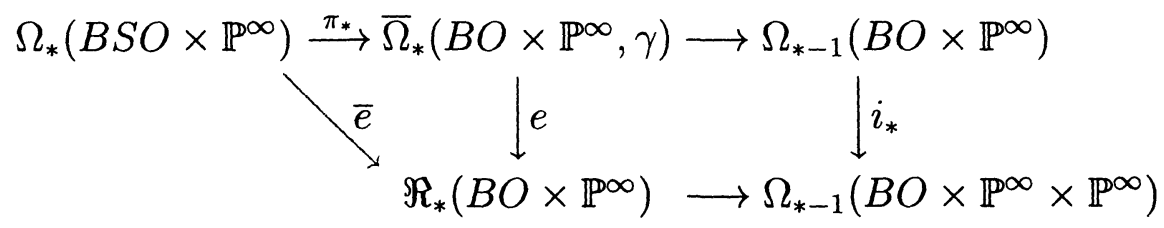

where $\bar{\rho}$ is the natural forgetful homomorphism, $i_{*}$ is the injection induced by the natural inclusion and $\pi_{*}$ is induced by the projection. The top line is the exact Gysin sequence corresponding to the orientation line bundle of $\gamma$ and the lower line is defined similarly 
by identifying $\Re_{*}\left(B O \times \mathbb{P}^{\infty}\right)$ with $\bar{\Omega}_{*}\left(B O \times \mathbb{P}^{\infty} \times \mathbb{P}^{\infty}, \lambda+\gamma\right)$ (see 9.21(ii) of [6]).

Now let $x \in \bar{\Omega}_{*}\left(B O \times \mathbb{P}^{\infty}, \gamma\right)$ be a 2-primary torsion so that $\rho(x)=$ 0 . To prove $x=0$ we first conclude from the exactness of the top line that there exists $y \in \Omega_{*}\left(B S O \times \mathbb{P}^{\infty}\right)$ with $\pi_{*}(y)=x$. Since $\bar{\rho}$ is injective restricted to the 2-primary torsion part of $\Omega_{*}\left(B S O \times \mathbb{P}^{\infty}\right)$, it suffices to show that $y$ is a 2-primary torsion. For this, let us consider the homomorphism

$$
d: \bar{\Omega}_{*}\left(B O \times \mathbb{P}^{\infty}, \gamma\right) \longrightarrow \Omega_{*}\left(B S O \times \mathbb{P}^{\infty}\right)
$$

defined by taking double covers. By definition $d \circ \pi_{*}=i d+T$ where $T$ is the involution on $\Omega_{*}\left(B S O \times \mathbb{P}^{\infty}\right)$ induced by $t$ and $t$ is the involution on $B S O$ defined by interchanging the two sheets. Since the involution on $H_{*}(B S O, \mathbb{Z})$ induced by $t$ is the identity, so is the involution $T$. It follows that $2 y=d \circ \pi_{*}(y)=d(x)$, and hence $y$ itself, is a 2-primary torsion as desired.

This completes the proof of our main theorem for the oriented cases.

\section{REFERENCES}

[1] M.F. Atiyah, Bordism and cobordism, Proc. Camb. Phil. Soc., 57 (1961), 200-208.

[2] J.M. Boardman, Singularities of differentiable maps, Publ. Math. I.H.E.S., 33 (1967), 21-57.

[3] R.L.W. Brown, Stiefel-Whitney numbers and maps cobordant to embeddings, Proc. Amer. Math. Soc., 48 (1975), 245-250.

[4] P.E. Conner, E.E. Floyd, Differentiable Periodic Maps, Springer-Verlag, Berlin, 1964.

[5] S. Feit, $k$-mersions of manifolds, Acta Math., 122 (1969), 173-195.

[6] U. Koschorke, Vector Fields and Other Vector Bundle Morphisms - a Singularity Approach, Lect. Notes in Math., vol.847, Springer-Verlag, Berlin and New York, 1981.

[7] G.-S. Li, On immersions in bordism classes, Math. Ann., 291 (1991), 373-382.

[8] C. Olk, Immersionen von Mannigfaltigkeiten in Euklidische Räume, Dissertation, Siegen, 1980.

[9] R.E.Stong, Cobordism of maps, Topology, 5 (1966), 245-258. 
Received April 2, 1992, revised July 9, 1993 and accepted for publication August 19, 1993.

Institute of Systems Science

ACADEMIa Sinica

BeIJING 100080, ChINA 




\section{PACIFIC JOURNAL OF MATHEMATICS}

Volume $166 \quad$ No. $2 \quad$ December 1994

Geometric aspects of Bäcklund transformations of Weingarten

submanifolds

STEVEN BUYSKE

Multipliers between invariant subspaces of the backward shift

225

ROBERT BRUCE CROFOOT

The Cauchy integral, analytic capacity and subsets of quasicircles

XIANG FANG

The number of lattice points within a contour and visible from the origin 295

Douglas Austin Hensley

On flatness of the Coxeter graph $E_{8}$

305

MASAKI IZUMI

Immersions up to joint-bordism

GUI SONG LI

Generalization of the Hilbert metric to the space of positive definite matrices

CARlangelo Liverani and Maciej WojtKowski

Periodicity, genera and Alexander polynomials of knots

SWATEE NAIK

On divisors of sums of integers. $\mathrm{V}$

ANDRÁS SÁRKÖZY and CAMERON LEIGH STEWART

Approximately inner automorphisms on inclusions of type $\mathrm{III}_{\lambda}$-factors

CARL WINSLøW

Correction to: "A convexity theorem for semisimple symmetric spaces"

KARL-HERMANN NEEB

Correction to: "Periodic points on nilmanifolds and solvmanifolds"

EDWARD KEPPELMANN

Correction to: "Partially measurable sets in measure spaces" 